\title{
Ranolazine Attenuates Stretch-induced Modifications of Electrophysiological Characteristics in HL-1 cells
}

\author{
Irene Del-Canto ${ }^{1,2}$, Lidia Gómez-Cid ${ }^{3}$, Ismael Hernández-Romero ${ }^{3}$, María S Guillem², María Eugenia \\ Fernández-Santos $^{3}$, Luis Such ${ }^{1}$, Francisco Fernández-Avilés ${ }^{3}$, Felipe Atienza ${ }^{3}$, Francisco J Chorro ${ }^{1}$, \\ Andreu M Climent ${ }^{3}$ \\ ${ }^{1}$ Instituto de Investigación Sanitaria INCLIVA, CIBERCV, Valencia, Spain \\ ${ }^{2}$ Universitat Politècnica de València, Valencia, Spain \\ ${ }^{3}$ Instituto de Investigación Sanitaria Gregorio Marañón, CIBERCV, Madrid, Spain
}

\begin{abstract}
Mechanical stretch induces an increase in $\mathrm{Na}^{+}$influx into myocytes, related to mechanisms including stretchactivated channels or activation of $\mathrm{Na}+/ \mathrm{H}+$ exchanger, involving changes in electrophysiological properties favouring arrhythmia induction. The aim of this study is to investigate the effects of mechanical stretch in cultured atrial murine cells (HL-1 myocytes) and its pharmacological response to ranolazine.

Confluent HL-1 myocytes with spontaneous fibrillatory activity was cultured in silicone membrane plates, and were stretched to $110 \%$ of resting length. The response to stretch under control conditions and under ranolazine effects was analyzed using a high-resolution optical mapping system.

HL-1 cells stretching increased atrial fibrillation dominant frequency in control conditions. The administration of ranolazine reduced this stretch-induced effect, and slowed the arrhythmia in baseline conditions.

Ranolazine attenuates the modifications of electrophysiological effects induced by myocardial stretch in HL-1 cells model of AF.
\end{abstract}

\section{Introduction}

Mechanical stretch is the common final stimulus to atrial myocytes in various cardiovascular diseases that are associated with atrial fibrillation (AF), such as mitral valvular disease, hypertension, and congestive heart failure [1]. Mechanical stretch induces an increase in $\mathrm{Na}^{+}$ influx in myocytes, related to mechanisms including stretch-activated channels or activation of $\mathrm{Na}^{+} / \mathrm{H}^{+}$ exchanger, and activates the reverse mode of $\mathrm{Na}^{+} / \mathrm{Ca}^{2+}$ exchanger, involving changes in electrophysiological properties favouring arrhythmia induction [2,3]. An increase in $\mathrm{Na}^{+}$current due to an increased number of active channels has also described as an expression of mechanosensitivity of the voltage-gated sodium channel $\mathrm{Na}_{\mathrm{v}} 1.5$ [4], which activation could enhance the persistent or late sodium inflow $\left(\mathrm{I}_{\mathrm{NaL}}\right)$ through the plateau of the action potential [5]. Moreover, $\mathrm{I}_{\mathrm{NaL}}$ current is also activated by the stretch-induced increase of intracellular $\mathrm{Ca}^{2+}$ concentration [5].

Ranolazine is an antiaginal agent that has been shown to exert a protective effect against cardiac arrhythmias linked to the enhancement of $\mathrm{I}_{\mathrm{NaL}}$ current and cellular $\mathrm{Ca}^{2+}$ overload [6]. The effects of ranolazine and the reduction of $\mathrm{Na}^{+}$entry into myocytes could modulate the electrophysiological effects of stretch.

The purpose of this work is to investigate the effects of mechanical stretch on electrophysiological characteristics during atrial fibrillation in cultured atrial myocyte monolayer in order to assess the effect of ranolazine and to determine whether it could modify the electrophysiological effects of stretch.

\section{Material and methods}

\subsection{Cell culture}

HL-1 cells were maintained, grown and proliferated according to the standard protocol established by Claycomb et al. [7] in 2x2 flexible PDMS wells.

\subsection{Experimental protocol}

After achieving further cell confluency, HL-1 cells spontaneous presented fibrillatory activity [8]. It has been reported that HL-1 cells are a viable in vitro model for study of the effects of mechanical stimulation on electrical characteristics [1]. In order to evaluate the effects of mechanical stretch on AF characteristics, myocytes were stretched in a strain unit to $110 \%$ of resting length for 10 minutes, and after this period stretch 
was supressed (Fig. 1). For each situation (basal, stretch, post-stretch), optical mapping recordings of fibrillatory activity were acquired under control conditions and after the administration of ranolazine $(50 \mu \mathrm{M}$ in Tyrode solution).
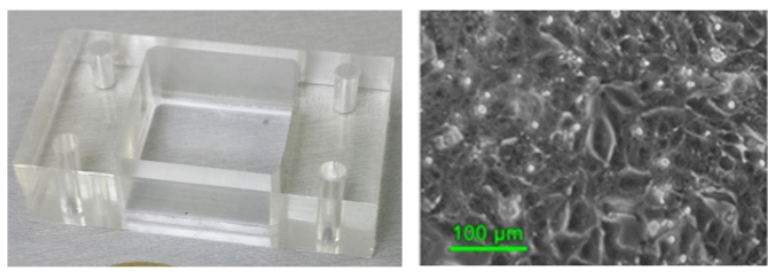

Figure 1. Confluent HL-1 murine monolayer in $2 \times 2$ polysimethylsiloxane (PDMS) membrane plates

\subsection{Optical mapping}

Calcium transient (CaT) imaging was developed by means of a high-resolution optical mapping system, and results were further analyzed by custom software developed in MATLAB [8].

Power spectra of optical signals were estimated by using Welch periodogram (2-s Hamming window overlap). Dominant frequency (DF) of each pixel was determined as the frequency with the largest peak in the spectrum between 0.05 and $30 \mathrm{~Hz}$. For each individual cell culture, the highest DF was defined as the maximum DF of the entire dish.

\subsection{Statistical analysis}

The previously analysis was performed in 12 monolayers of fibrillating HL-1 cells. Data are presented as mean \pm standard deviation. Continuous variables were compared using repeated measures ANOVA test or Wilcoxon and Friedman's tests, depending on the variable's statistical distribution.

\section{Results}

In all 12 analyzed cell cultures, AF was stable along the protocol duration. CaT maps of a representative example of each situation are shown in the figure 2 .

\subsection{Effects of ranolazine on baseline activation patterns during $\mathbf{A F}$}

During baseline recordings (prior to stretch) dominant frequency was significantly smaller under ranolazine perfusion than in control conditions $(3.04 \pm 0.18 \mathrm{~Hz}$ vs. $3.65 \pm 0.20 \mathrm{~Hz}, \mathrm{p}<0.01$ ) (Fig. 3), where the slowing effect of the drug is evident. After supressing stretch, ranolazine reduces frequency activation slowing the arrhythmia $(3.00 \pm 0.24 \mathrm{~Hz}$ vs. $3.63 \pm 0.31 \mathrm{~Hz}, \mathrm{p}<0.05)$ (Fig. 3).

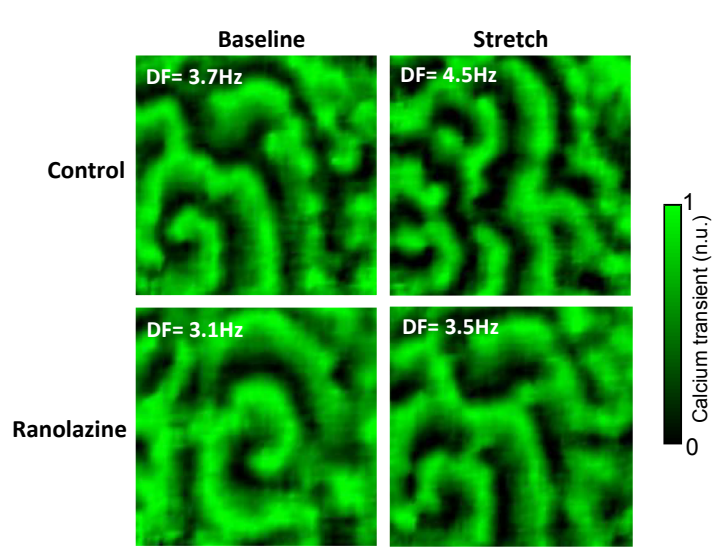

Figure 2. CaT maps of representative cell cultures in basal (left) and stretch (right) situations, during control conditions (top) and after the administration of ranolazine (bottom).

\subsection{Effects of ranolazine on stretch- induced modifications of activation patterns during AF}

In control series, DF increased significantly during stretch $(3.65 \pm 0.20 \mathrm{~Hz}$ vs. $4.35 \pm 0.23 \mathrm{~Hz})$ and returned to baseline values after stretch suppression $(3.63 \pm 0.31$ $\mathrm{Hz}$ ) (Figure 3). The magnitude of stretch-induced DF increment was $19.7 \%$.

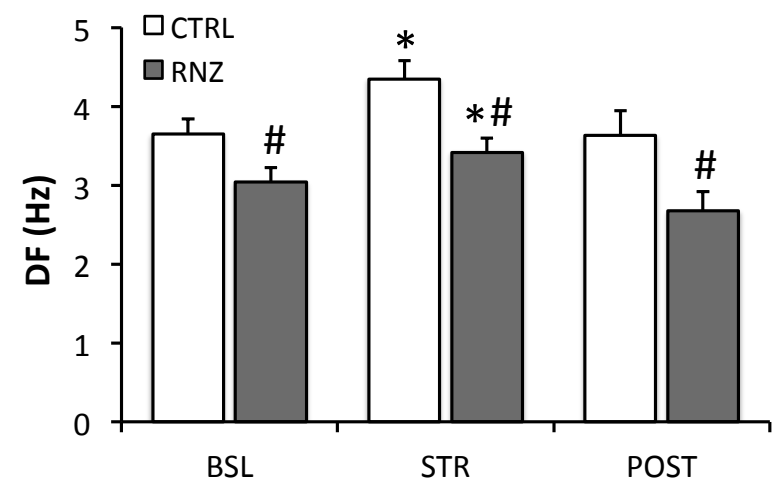

Figure 3. Dominant frequency in control and ranolazine conditions, for basal, stretch and post-stretch situations. *p vs. BSL, \#p vs. CTRL.

The administration of ranolazine attenuated the electrophysiological effects of stretch. Under ranolazine action, stretch-induced DF increasing remained (3.04 \pm $0.18 \mathrm{~Hz}$ vs. $3.42 \pm 0.18 \mathrm{~Hz}, \mathrm{p}<0.05$ ), but was reduced (Fig. 3). In fact, the magnitude of DF increment was smaller under ranolazine effects than in control conditions (12.4\% vs. 19.7\%, p<0.01) (Fig. 4).

Moreover, stretched cell cultures treated with ranolazine presented lower dominant frequencies $(3.42 \pm$ $0.18 \mathrm{~Hz}$ vs. $4.35 \pm 0.23 \mathrm{~Hz}, \mathrm{p}<0.01$ ) (Fig. 3). 


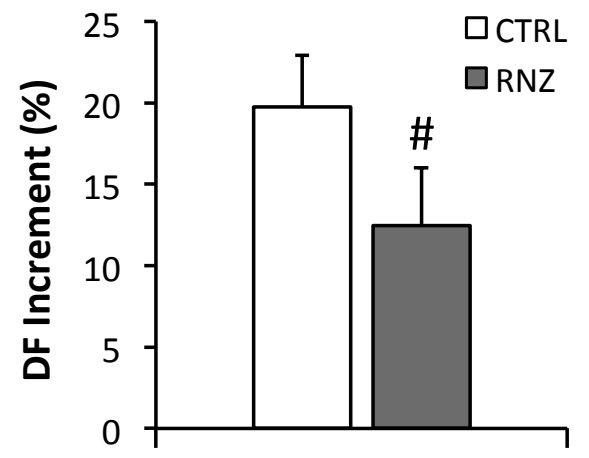

Figure 4. Increment of dominant frequency in control and ranolazine conditions after stretching. $\# \mathrm{p}<0.01$ vs. CTRL.

\section{Discussion}

In the present study, we have used an experimental model involving HL-1 cells monolayer in which the analysis of the spectral characteristics of myocardial activation during atrial fibrillation allow us to track the time course of the electrophysiological changes induced by stretch. The study was carried out to analyze the effects of ranolazine on the electrophysiological responses induced by mechanical stretch.

In the absence of stretch, we observed a slowing effect on activation during AF by ranolazine. Dominant frequency was lower under drug action, effect associated to an increase in refractoriness. In this situation, rather than by block of late $\mathrm{Na}^{+}$current which would shorten repolarization, the increase of refractoriness would be due to block of repolarizing $\mathrm{I}_{\mathrm{Kr}}$, known effect for ranolazine (IC50 $\approx 10 \mu \mathrm{M})$.

In the present work, as in previous studies on isolated Langendorff-perfused rabbit hearts [9], we found that stretch increased the AF dominant frequency, which can be considered as proarrhythmic.

The stretch-induced changes on HL-1 activation were attenuated under the action of ranolazine. Dominant frequency increments under stretch were smaller under ranolazine effect and we observed a reduction in the activation rate, which can be considered as antiarrhythmic. These results support the hypothesis that this $\mathrm{I}_{\mathrm{NaL}}$ blocker could modulate cardiomyocyte mechanosensitivity and suggest that other protective effects of ranolazine may be involved.

A factor that may be related to the effects of ranolazine during HL-1 cells stretch is the action of the drug upon the stretch-induced modulation of $\mathrm{Na}_{\mathrm{v}}$ 1.5. The mechanosensitivity of $\mathrm{Na}_{\mathrm{v}} 1.5$ consists of accelerated kinetics, an increased peak current, and stabilization of inactivation [1]. In fact, modulation of mechanosensitivity of $\mathrm{Na}_{\mathrm{v}} 1.5$ has been demonstrated at high ranolazine concentrations $(50 \mu \mathrm{M})$, though the drug effects depend on the magnitude of stretch and on the ranolazine membrane concentration [1].
Finally, aside from $\mathrm{Na}_{\mathrm{v}} 1.5$ and $\mathrm{I}_{\mathrm{NaL}}$, ranolazine inhibits other ionic currents as $\mathrm{I}_{\mathrm{CaL}}$ and $\mathrm{I}_{\mathrm{Na}-\mathrm{Ca}}$, which are some of the mechanisms involved in the effects of stretch $[6,9]$. Nevertheless, the inhibition of these currents was weak at atrial level for the concentration used in this work [6].

\section{Conclusion}

Ranolazine attenuates the electrophysiological effects responsible for the acceleration of $\mathrm{AF}$ induced by myocardial stretch in HL-1 cells.

\section{Acnowledgements}

Supported by grants from the Instituto de Salud Carlos III: Grants CB16/11/00486, CB16/11/00292, PI13-01882 PI14/00857, PI15/01408, PI14/00857, PI16/01123, TEC2013-46067-R, DTS16/0160 and IJCI-2014-22178 by Spanish Ministries and cofound with FEDER founds.

\section{References}

[1] Strege P, Beyder A, Bernard C, et al. Ranolazine inhibits shear sensitivity of endogenous $\mathrm{Na}^{+}$current and spontaneous action potentials in HL-1 cells. Channels 2012;6:457-62.

[2] Cingolani HE, Perez NG, Cingolani OH, et al. The Anrep effect: 100 years later. Am J Physiol Heart Circ Physiol 2013;304:H175-82.

[3] Ravelli $F$, Masè $M$, del-Greco $M$, et al. Acute atrial dilatation slows conduction and increases AF vulnerability in the human atrium. J Cardiovasc Electrophysiol. 2011;22:394-401.

[4] Peyronnet R, Nerbonne J, Kohl P. Cardiac mechano-gated ion channels and arrhythmias. Circ Res 2016;118: 311-29.

[5] Maltsev VA, Undrovinas A. Late sodium current in failing heart: friend or foe? Prog Biophys Mol Biol 2008;96:42151.

[6] Antzelevitch C, Burashnikov A, Sicouri S, et al. Electrophysiological basis for the antiarrhythmic actions of ranolazine. Heart Rhythm 2011;8:1281-90.

[7] Claycomb WC, Lanson NA, Stallworth BS, et al. HL-1 cells: a cardiac muscle cell line that contracts and retains phenotypic characteristics of the adult cardiomyocyte. Proc Natl Acad Sci USA1998;95:2979-84.

[8] Climent AM, Guillem MS, Fuentes L, et al. Role of atrial tissue remodeling on rotor dynamics: an in vitro study. Am J Physiol Heart Circ Physiol 2015;309:H1964-73.

[9] Del-Canto I, Such-Miquel L, Brines L, et al. Effects of JTV-519 on stretch-induced manifestations of mechanoelectric feedback. Clin Exp Pharmacol Physiol 2016;43:1062-70.

Address for correspondence.

Andreu M. Climent.

Instituto de Investigación Sanitaria Gregorio Marañón.

Hospital GU Gregorio Marañón. Edificio Materno Infantil.

c/ O’Donnell 48, 28009, Madrid, Spain

acliment@cibercv.es 
Page 4 